\begin{tabular}{cl}
$\begin{array}{c}\text { STUDI } \\
\text { FRANCESI }\end{array}$ & $\begin{array}{l}\text { Studi Francesi } \\
\text { Rivista quadrimestrale fondata da Franco Simone } \\
\end{array}$ \\
\cline { 1 - 2 } & $\begin{array}{l}\mathbf{1 8 6} \text { (LXII | III) } \mid \mathbf{2 0 1 8} \\
\text { Varia }\end{array}$ \\
\end{tabular}

\title{
Michel Butor, Au temps du noir et blanc
}

\section{Anna Bucarelli}

\section{OpenEdition \\ Journals}

\section{Edizione digitale}

URL: http://journals.openedition.org/studifrancesi/15835

DOI: 10.4000/studifrancesi.15835

ISSN: 2421-5856

\section{Editore}

Rosenberg \& Sellier

\section{Edizione cartacea}

Data di pubblicazione: 1 décembre 2018

Paginazione: 530-531

ISSN: 0039-2944

\section{Notizia bibliografica digitale}

Anna Bucarelli, «Mıchel Butor, Au temps du noir et blanc», Studi Francesi [Online], 186 (LXII | III) | 2018, online dal 01 janvier 2019, consultato il 06 janvier 2021. URL: http://journals.openedition.org/ studifrancesi/15835 ; DOI: https://doi.org/10.4000/studifrancesi.15835

Questo documento è stato generato automaticamente il 6 janvier 2021.

\section{(c) (†)}

Studi Francesi è distribuita con Licenza Creative Commons Attribuzione - Non commerciale - Non opere derivate 4.0 Internazionale. 


\title{
Michel BUTOR, Au temps du noir et blanc
}

\author{
Anna Bucarelli
}

\section{NOTIZIA}

MicheL ButOR, Au temps du noir et blanc, introduction de Mireille Calle-Gruber, Paris, Delpire Éditeur, 2017, «Des images et des mots», 312 pp.

1 Voluto da Michel Butor e portato a termine da Mireille Calle-Gruber con il sostegno delle figlie dello scrittore, che le hanno dato accesso all'archivio famigliare, il progetto di questo volume trae origine-come si legge nell'introduzione-«d'un geste de reconnaissance intime: celui du vieil écrivain chevronné donnant la main, au bord de sa quatre-vingt-dixième année et après une carrière vertigineuse, au débutant de vingtquatre ans qu'il avait été» (M. CALLE-GRUBER, «Quand Michel Butor photographiait le Génie du lieu. Un écrivain à la fenêtre des images», pp.7-18; p.8). Un gesto di riconoscimento che è anche e innanzitutto svelamento di una dimensione pressoché sconosciuta della produzione butoriana, quella del giovane fotografo che, tra il $1951 \mathrm{e} \mathrm{il}$ 1961, si dedica «assidûment, intensément» (p. 7) all'arte delle immagini in bianco e nero con la sua Semflex, versione francese della storica Rolleiflex. Dieci anni in cui le fotografie si moltiplicano al pari dei viaggi e della scrittura letteraria, dal primo progetto di Passage de Milan fino all'apparizione di Mobile. Étude pour une présentation des États-Unis (1962) che segna il definitivo abbandono dell'attività fotografica e dei codici romanzeschi in favore di inedite forme di scrittura e collaborazioni artistiche volte a «activer l'un par l'autre les potentiels de l'art du texte et de l'art de l'image» (p. 11). Di questa produzione giovanile il volume propone centodiciannove fotografie selezionate dallo stesso Butor con la collaborazione di Mireille Calle-Gruber. Sono immagini en noir et blanc scattate in Francia, Turchia, Gran Bretagna, Italia, Grecia, Spagna e Stati Uniti che invitano lo sguardo a soffermarsi su elementi archeologici e architettonici (chiese, templi, rovine, case, grattacieli, mura, balconi, portici, colonne), oggetti e luoghi di vita 
quotidiana (barche, tende, cancelli, inferriate, edicole, piazze, vie), qualche paesaggio (urbano, marino, rurale e naturale) e alcune figure umane - «silhouettes passantes, énigmatiques» (p.16) - raramente protagoniste e per lo più anonime (dei bambini, un pastore, un guardiano, una ragazza), con la sola eccezione di Georges Perros - l'amico del cuore-e della moglie Tania (p. 144). Scatti composti pazientemente, minuziosamente, come vuole la stessa fotocamera usata («Le format carré, l'attitude à laquelle oblige le reflex, ne pouvaient que m'encourager à des images minutieusement, patiemment composées», p. 286) e d'accordo con la contrapposizione butoriana tra la «photo aventureuse» della Leica - colta al volo con un solo occhio nel mirino - e la «photo méditative» della Rolleiflex, che si ottiene con «l'appareil sur son estomac, les deux yeux impliqués dans la mise au point, le cadrage» (M. ButoR, «Philosophie du polaroïd», p. 275). Senza dimenticare che il reflex - con il suo meccanismo a specchio suggerisce la riflessività stessa della sua arte fotografica: «l'image ne doit pas oublier le processus de son invention. Ouverture - de l'obturateur, de la paupière, de la pupille. Découpe - d'un cadre, une scène, une architecture, un dessin. Exposition - à la lumière et aux ombres, aux éclats de jour qui donnent formes ou déforment» (p. 14). Non si tratta, per Butor, di rappresentare semplicemente un luogo, bensì di «inventer une image» (p. 286) per rappresentare il génie du lieu che di lì a poco designerà un nuovo genere letterario (la pubblicazione del Génie du lieu è del 1958). Ciò significa, osserva Calle-Gruber (p. 15), lasciarsi impregnare dalle energie del luogo, farsene ricettacolo e, nello stesso tempo, farsi osservatore attento, acuto ( 'J'avais besoin non seulement de me fondre dans le paysage, mais de revenir sur les lieux, de supputer la lumière dont j'avais besoin pour les scènes que je voulais capter dans ces théâtres»; «Le viseur était un instrument d'exploration qui me faisait voir ce que je n'aurais pas vu sans lui», p. 286). In altre parole, essere al contempo «lui-même corps photographique et matière de rêves» (p. 15).

2 Al riconoscimento e svelamento dell'opera del giovane fotografo si accompagna nel volume il gesto di creazione dello scrittore ottuagenario che compone il materiale secondo un ordine non meramente o solo apparentemente cronologico in cui si ritrovano «son goût des séries, des variantes, des duplications et univers symétriques, son art du contrepoint, son don d'ubiquité» (p. 17). Alcuni esempi: la ripresa variata del motivo dei Bateaux o degli Stores, le immagini riproposte a poche pagine di distanza in una più ampia o rivelatrice prospettiva come la cupola e le colonne di Granada (pp. 123 e 131, 125 e 132). Dei testi che Butor avrebbe dovuto comporre per accompagnare le fotografie non resta, invece, che una pagina introduttiva sulla sua attività di fotografo (p.21) e qualche strofa manoscritta di cui il volume serba una traccia fotografica (p. 260). Al loro posto, frammenti di descrizioni, ekphrasis, racconti, poesie, dialoghi e altri generi ibridi tratti dall'opera di Michel Butor (con due inediti), da Mobile e Réseau aérien del 1962 a due testi - un inedito e un livre d'artiste - del 2016. La scelta di queste citazioni da parte della curatrice si ispira, oltre che alle immagini stesse (come $\mathrm{i}$ frammenti del Génie du lieu relativi ai luoghi fotografati), alla grammatica del montaggio butoriano (p.17). Le pagine raccolte nella parte finale del volume «Sur la photographie» (pp. 261-302) sono testi pubblicati nel corso di più di un ventennio $\mathrm{e}$ successivamente raccolti nel volume X delle Euvres complètes curate da M. Calle-Gruber: «Alchimigramme» (1991), «pour Pierre Cordier» (pp. 263-267); «Du monochrome en photographie» (2004), «pour Michèle Auer e Gérard Lüthi» (pp. 269-274); «Philosophie du polaroïd» (2007), «pour Joël Leick» (pp. 275-277); «Poésie et photographie» (1984), «pour André Villers» (pp. 279-283) e «Souvenirs photographiques. Un viseur dans la 
tête» (2002), "pour Philippe Lutz» (pp. 285-302). Testi che mostrano come all'attività del fotografo sia subentrata la scrittura «sur ou pour des photographes» (p. 287) e che ricollocano la pratica della fotografia nel più ampio contesto di un discorso critico e poetico che non solo a essa si lega indissolubilmente ma che per molti aspetti ne scaturisce. Come osserva la curatrice (p.13), la conoscenza dell'opera fotografica rovescia infatti il rapporto di priorità tra parola e immagine, sempre presente nella vasta quanto proteiforme opera di Butor, attribuendo alla «naissance de l'œil photographique» (p.13) un ruolo germinale nella genesi del Génie du lieu e dell'intera opera visionaria e polimorfa dello scrittore: «La réflexion/réflexivité que lui enseigne l'exercice photographique, il la transpose sur la page littéraire, où elle devient bientôt le principe philosophique de sa recherche, et de son rapport aux langues, aux êtres, aux choses» (p. 16). 